\title{
ESPER 2, an improvised oscilloscope music set
}

\author{
Eric Lennartson
}

USA

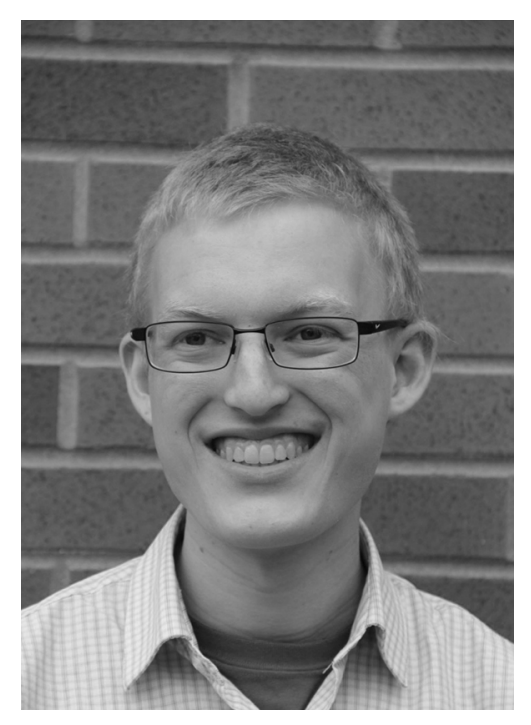

Eric Lennartson is a composer, improviser, and oscilloscope artist based in Los Angeles. He received his Bachelors in percussion performance from the University of North Texas and his Masters in performance and composition at the California Institute of the Arts. His audio-visual work uses old analog oscilloscopes to create non objective imagery that interacts with dense noise, pulsating tones, and unstable beats. As a result, sound and image create a feedback loop of meaning. Through this interaction he explores the different perspectives and meaning inherent to the sound and image itself.

E-mail: lennartsoneric@gmail.com

Link to "ESPER 2": https://youtu.be/Mc7PE1CKtbw 


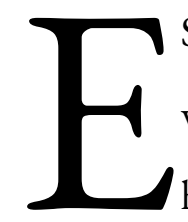

SPER 2 is something known as Oscilloscope Music, or Vector Synthesis. It is a unique art form where specialized digital sounds create music and visuals. To do so, a stereo audio signal controls horizontal and vertical movement. The left channel controls horizontal movement and the right channel controls vertical movement. It is similar to an Etch a Sketch, but if the knobs could move at the audio rate.

One interesting aspect of oscilloscope music is its disregard for most of the usual music production techniques. Things that are usually useful such as compression, mixing, and panning become useless. The changes in the sound that such techniques produce result in messier visuals. For me, the joy is coming up with a visual and figuring out what that means for audio synthesis. From there, I can begin exploring the new system I have created.

Although the restriction of sound and image always having a 1:1 ratio is rather austere, there are actually many possibilities. Most visuals have more than one way of being produced. Meaning that there are many sounds for the same visual. Often, a good patch is one that can create the same visual in more than one way. This opens up the sonic possibilities, while maintaining the 1:1 ratio of sound to image. Because of this, nothing ever needs to stay as it is, and each piece can be bound by its own set of rules. There are some general principles at play, but each patch is usually unique. It need not follow the rules or design from the last patch.

In general, oscilloscope music sits between genres. Because of this, I have had ESPER 2 act as a live set where I am navigating the patch in real time. It is usually performed at a venue with many other people watching the work projected onto a screen. It has also acted as an installation, with the sound turned off. In this form, the work focuses more on the visuals and historical importance of the oscilloscope. As well as it's references to early computing.

For ESPER 2, the Pure Data patch itself is both code to create oscilloscope music, and an instrument. While this is quite a clunky way of doing things (there are de2finitely easier ways to do it) the result can be useful. A consequence of this design choice is that only one parameter or effect can change at a time. The result is a piece that takes on a simple organic form that is both didactic and exploratory. The interest for me is in investigating the various combinations of parameters. I am always asking, how does this system I've created operate? After some time I gain a feeling for what it was that I have made. From this, I am able to create a loose framework to improvise around. In ESPER 2 I decided upon certain events, but how I got there, and all other details were left to the moment. 